\title{
Combinaciones de marcadores del discurso en el lenguaje hablado: un estudio empírico de anda, vamos, vaya y venga
}

\author{
Co-occurrences of Discourse Markers \\ in Spoken Language: An Empirical Study \\ of anda, vamos, vaya and venga
}

SANNE TANGHE

Departamento de Lingüística

Universidad de Gante

Blandijnberg 2. Gent, 9000. Bélgica

sanne.tanghe@ugent.be

Orcid ID: 0000-0001-5654-9981

Resumen: En este trabajo se abordan las combinaciones de dos marcadores en el lenguaje hablado a partir del análisis de los marcadores derivados de verbos de movimiento en español (anda, vamos, vaya y venga). En una primera fase se propone un método cuantitativo para identificar las combinaciones no casuales calculando la fuerza de asociación entre sus constituyentes. Dentro del grupo de las combinaciones no casuales y con base en un análisis cualitativo es posible distinguir tres tipos según su estructura interna y la correspondiente integración funcional de sus constituyentes. En cuanto a las intenciones comunicativas de las combinaciones, el estudio muestra que son ante todo sumativas. La combinación pero vamos es la única combinación que parece haber desarrollado un valor holístico dada su reducción de composicionalidad al nivel semántico. Por su alta frecuencia de caso y por su emparejamiento formalfuncional cuando se usa para ceder el turno, concluimos que pero vamos es una combinación bien sedimentada en la mente de los hablantes.

Palabras clave: Marcadores del discurso. Combinaciones. Lenguaje hablado. Estudio de corpus. Verbos de movimiento.
RECIBIDO: 7 DE JULIO DE 2016 ACEPTADO: 14 DE SEPTIEMBRE DE 2016
Abstract: In this study the co-occurrences of two markers in spoken language are addressed by analyzing the markers derived from motion verbs in Spanish (anda, vamos, vaya y venga). First of all, a quantitative method is proposed to identify the non-casual co-occurrences. By calculating the strength of association between the constituents the casual $\mathrm{co}^{-}$ occurrences can be distinguished from the true ones. Within this last group, and on the basis of a qualitative analysis, three types of co-occurrence can be discerned depending on their internal structure and the corresponding functional integration of their constituents. Concerning the communicative intentions of the co-occurrence, the study shows that they are above all summative Pero vamos is the only co-occurrence that seems to have developed a holistic meaning, given its reduction in compositionality on the semantic level. Because of its high case frequency and its form-function pairing when used as a turn-yielder, we conclude that pero vamos is a wellentrenched combination in the minds of the speakers.

Keywords: Discourse Markers. Co-occurrences. Spoken Language. Corpus Study. Verbs of Motion. 


\section{INTRODUCCIÓN}

E n la amplia bibliografía sobre los marcadores del discurso predominan los estudios que se centran en las funciones que desempeñan y en las diferencias entre y/o similitudes en los usos de formas diferentes. Menos atención ha recibido el análisis de los marcadores en el eje sintagmático. Por eso, en este artículo nos dedicamos al estudio de las secuencias de dos (o más) marcadores en el flujo de habla. Más en particular, nos interesan las combinaciones de esos marcadores conversacionales que pueden usarse individualmente (pero, va, vamos, etc.), al contrario de las así llamadas locuciones marcadoras (Ruiz Gurillo), que se forman a partir de elementos léxicos y cuyos constituyentes no necesariamente se emplean como marcadores en sí (por lo visto, de todos modos, etc.).

El primer objetivo del presente estudio es determinar el grado de convencionalización de las combinaciones como unidades fijas. ${ }^{1}$ La cuestión de la casualidad o de la posible fijación de las combinaciones de marcadores ha sido abordada desde varias perspectivas. Dostie (2013) propone estudiar las combinaciones de forma análoga a las asociaciones sintagmáticas de las palabras pertenecientes a las clases gramaticales 'tradicionales' (sustantivos, verbos, adjetivos, etc.). Aplica, así, las nociones del dominio de la lexicografía a un fenómeno pragmático; basándose en la interdependencia pragmática de los constituyentes, distingue para el francés de Quebec combinaciones discursivas libres (bien vois-tu), colocaciones discursivas (voyons donc) y locuciones discursivas (bon ben). Los tres tipos se sitúan en un continuo, siendo aquellas las que carecen de dependencia pragmática mutua entre sus constituyentes, mientras que estas representan las unidades semánticas fijas.

Un enfoque similar es el de Cuenca y Marín que se basa en la interdependencia pragmática de los marcadores para determinar así diferentes grados de integración formal y funcional de los constituyentes, a saber, distinguen entre la yuxtaposición (y mientras), la adición (y bueno) y la composición (pues vale). Al lado del criterio semántico-pragmático, describen las combinaciones según los tipos de marcadores que comprenden (conjunciones, conectores parentéticos, conectores pragmáticos o interjecciones). Así, por ejemplo, la combinación de dos conjunciones (y mientras) es un ejemplo prototípico de la yux-

1. Optamos por el término combinación por su neutralidad en comparación con otros términos, como fórmulas conversacionales o asociaciones, que parecen sugerir cierta afinidad entre sus constituyentes. 
taposición, ya que los constituyentes tienen una función semántica y sintáctica distinta.

Por último, el enfoque de Pons Bordería es, antes bien, estructural, ya que analiza las combinaciones de marcadores dentro del marco teórico de una teoría de unidades de la conversación. Concluye que cuando existe una frontera de unidad entre los dos constituyentes (bueno pues), el primer marcador tiene ámbito sobre el segundo, mientras que una combinación de dos marcadores está lexicalizada cuando los constituyentes pertenecen a la misma unidad estructural (pues bueno).

En este artículo se propone un método cuantitativo para abordar la cuestión del grado de fijación sintagmática de las combinaciones. Concretamente, se determina el grado de asociación entre los constituyentes de las combinaciones aplicando una medida de asociación que es un método transferido del dominio de las colocaciones léxicas (Evert). Este método permite redactar un ranking de las combinaciones según la fuerza de asociación entre sus dos constituyentes (sección 3) con las combinaciones más casuales (o menos fijas) situadas a la cola de lista.

Además de estudiar el grado de fijación de las combinaciones, nos centraremos en la hipótesis de que las combinaciones tienen un significado antes ‘sumativo' que 'holístico' (Mosegaard Hansen 1998, 233; Waltereit 104). Según un estudio de Waltereit (104) sobre combinaciones con los marcadores ben y bon en francés "leur lexicalisation [de las combinaciones] conserve les sémantismes des deux marqueurs concernés au lieu de les amalgamer dans un nouveau sens unique". Con base en un estudio de las funciones de las combinaciones y de sus constituyentes en un corpus de lenguaje hablado, pretendemos verificar la hipótesis de que en una combinación de marcadores cada uno de los constituyentes mantiene su significado individual.

Por su flexibilidad semántico-pragmática y sintáctica (Castillo Lluch), recurrimos a los marcadores derivados de verbos de movimiento. Concretamente, nos interesan las formas anda, vamos, vaya y venga que se yuxtaponen con otro marcador (pero vamos, pues vaya, etc.), que se combinan entre sí (anda ven$g a$ ) o que se reduplican (anda anda). En la sección 2 describimos las funciones de estos marcadores y presentamos el corpus que manejamos en el presente estudio. Luego, en la sección 3 se presenta y se aplica la metodología cuantitativa. La interpretación de estos resultados cuantitativos consiste en tres partes. En la sección 4.1 analizamos la estructura interna de las combinaciones, lo cual nos permitirá distinguir varios tipos de combinaciones. En la sección 4.2 se 
estudia el grado de integración funcional de los constituyentes, es decir, se examina si las combinaciones tienen un significado sumativo o si adquieren un valor holístico. La sección 4.3 está dedicada a un estudio de caso de la combinación que presenta la frecuencia de caso más alta, a saber: pero vamos.

\section{LOS MARCADORES DE VERBOS DE MOVIMIENTO}

Las formas verbales de movimiento que más frecuentemente se emplean como marcadores del discurso en el español peninsular son anda, vamos, vaya y venga. Estas formas se han recategorizado como marcadores convencionalizando cada vez más implicaturas conversacionales (Traugott/Dasher 80). El resultado de este proceso de cambio semántico-pragmático son formas con contenido, ante todo, procedimental, esto es, ayudan en el proceso de interpretación (Montolío Duran; Portolés). Este enriquecimiento pragmático de las formas conlleva cambios a nivel morfológico y sintáctico. Así, las formas usadas como marcadores no tienen estructura argumental (venga usted, un beso), presentan una gran libertad posicional (venga, un beso / Un beso, venga), han perdido las capacidades sintácticas de las formas originarias ('anda y vamos, a trabajar) y se han fijado morfológicamente (Castillo Lluch).

En el curso de este proceso de cambio, los usos pragmáticos de las formas se han ido diversificando y estratificando, por lo que los marcadores se caracterizan actualmente por un alto grado de polisemia (Mosegaard Hansen 2008; Hummel; Tanghe). Por razones heurísticas, clasificamos los valores pragmáticos de los marcadores que estudiamos en macrocategorías funcionales (Tanghe). Con base en estudios anteriores de los marcadores anda, vamos, vaya y venga (Fuentes Rodríguez 1998; Gras Manzano/Polanco Martínez/Santiago Barriendos; Castillo Lluch; Polanco Martínez; Tanghe) y en diccionarios especializados (Santos Río; Briz Gómez/Pons Bordería/Portolés; Fuentes Rodríguez 2009), se destacan tres clases de funciones pragmáticas de estas formas, a saber: la función apelativa, la expresiva y la metadiscursiva.

En primer lugar, los marcadores se emplean con función apelativa cuando el hablante se dirige al interlocutor e intenta persuadirlo para que se comporte, piense, etc. de cierta manera:

1) $<$ risas/ $>$ dame un piti anda (COLAm)

2) $<$ H1 $><$ ininteligible $><$ simultáneo $>$ Bien, entonces ya... vamos a darnos un poco de prisa, que si no no va a darnos tiempo a todos, y además hacer algunos comentarios que tengo que hacer. 
$<$ Hg $>$ Venga, vamos, dale. (CORLEC)

3) $<\mathrm{H} 2>$ ¿Qué quieres preguntarme? Venga. (CORLEC)

En el ejemplo (1) y (2) los marcadores refuerzan la orden expresada por el imperativo al que acompañan, respectivamente dame y dale. En el tercer ejemplo, el marcador venga anima al interlocutor a hacerle su pregunta. El marcador vaya carece de la posibilidad de tener una función apelativa. ${ }^{2}$

En segundo lugar, los cuatro marcadores cumplen una función expresiva cuando indican las actitudes o sentimientos del hablante para con una situación (extra)lingüística. Son varios los valores expresivos de los marcadores: indican asombro (4), disgusto (5), desacuerdo (6), asentimiento (7), etc.:

4) y este chico que hay aquí?/ era mi pretendiente P: ¡anda! (Val.Es.Co)

5) $<$ H1 > ¡Ah...! </simultáneo > y Julia que se divorcia y ¡menudos gastos!, ¡vaya...! Porque solamente pagar abogados, <simultáneo> ¡qué barbarida $<($ d $)>$ ! (CORLEC)

6) Enc. - ¿Escribe usted?

Inf.- ¡Vamos, no! Escribo cartas. Escribo a las amigas, claro, como no tengo otro medio de comunicación; (MC-NLCH)

7) $<\mathrm{H} 2>\dot{\mathrm{A} h}$, sí? Ay, me lo tienes que leer.

$<\mathrm{H} 1>$ ¿Te lo leo ahora?

$<H 2>$ Venga sí. Lo que pasa que teníamos que ir a... a tomar la sopa, para que no se enfríe. (CORLEC)

Por último, los marcadores adoptan una función metadiscursiva cuando se orientan hacia el discurso y se ocupan de su desarrollo o regulación. De esta manera, vamos y vaya se emplean para indicar una reformulación (8) (9), vamos tiene valor continuativo a fin de mantener el turno de habla (10), venga y (pero) vamos indican el cierre del discurso o el fin de un (sub)tema de conversación o una digresión (11) (12) y, finalmente, vamos se usa para subrayar la fuerza argumentativa de un enunciado (13):

8) B: pero a lo mejor tienes suerte para ti y no para los demás porque yo de mis hijos? y tengo cuatro / hay uno que tiene suerte / pero tiene suerte para él / vaya? no una suerte loca pero él [juega a la lotería=] (Val.Es.Co)

2. En cuanto al uso de vaya con valor apelativo, alega Octavio de Toledo y Huerta (54) que "el español (centro)americano parece haberlo conservado con mayor vigor". 
9) que me están ustedes diciendo que quiero perregaña eh perraña vamos (COLAm)

10) que... la corrida de Concha Navarro, que estaba arreglada, no para los toreros, no, para... y tienen vamos, tienen bastante prestigio como lo tenéis vosotros también, eh... (CORLEC)

11) *BLA: hhh //\$ bueno //\$ sí / un día o dos / como <mucho> //\$ \%act: (1) assent

*YOL: $[<]<$ supongo $>/ / \$$ venga $/ / \$$ pues nos vemos luego / vale ?\$

* BLA: venga $/ / \$$

${ }^{*}$ YOL: venga //\$

* BLA: $[<]$ venga $/ / \$$

* YOL: hasta <luego $>/ / \$(C-O R A L-R O M)$

12) Yo no sé, los hoteles estaban siempre llenos de sudamericanos. Claro, la misma frontera con Austria está bastante cerca de Viena, porque se tardan solo unas horas nada más en llegar. Aunque las carreteras no son buenas, pero vamos... Y hay mucho más turismo. Mientras que, en Polonia, no, por lo menos, el norte de Polonia es pobre, pobre, pobre al máximo, vamos. (MC-NLCH)

13) Entre un envase poco apropiado y un envase apropiado ¡no hay una diferencia! ¡Cuesta lo mismo hacer una cosa mal que hacerla bien! $<$ H6> Yo tampoco estoy de acuerdo con eso, vamos... <risas $>$. (CORLEC)

Como hemos dicho más arriba, por el proceso de cambio estas formas verbales han perdido sus capacidades sintácticas y como marcadores no pueden, por ello, coordinarse entre sí (*anda y venga). A pesar de ello, los marcadores objeto de estudio sí pueden formar combinaciones, esto es, pueden yuxtaponerse (14), iterarse (15) o combinarse con otros marcadores (16) (17), como ilustran los ejemplos siguientes:

14) Tiene que ser él (( )) ¿quiéen?... venga anda / ¿dónde estabas?\$ (Val.Es.Co)

15) Inf.- Siéntate, anda, anda. ¿Sabe usted que está bueno el este? Vamos a echar otra copita. (MC-NLCH)

16) *MAM: Ana el domingo / ayer / todo el día acostada //\$

*PAP: ¡jo! //\$

${ }^{*}$ MAM: con la gripe //\$

${ }^{*}$ PAP: pues vaya ...\$ (C-ORAL-ROM) 
17) ${ }^{*} \mathrm{CAR}$ : pues eso vas al notario / y le dices que te haga un poder / y [/] y ya con el poder notarial / sí que lo puedes recoger tú //\$ pero vamos que / si puede venir él mejor //\$ \&mm / un ratito / que se pase //\$ (C-ORAL-ROM)

El estudio actual examina estas combinaciones en un corpus que cubre una gran variedad de tipos textuales del lenguaje hablado (entrevistas, conversaciones coloquiales, etc.). Las bases de datos fuentes de este corpus son: Corpus integrado de referencia en lenguas romances (C-ORAL-ROM), Corpus Oral de Referencia del Español Contemporáneo (CORLEC), Macrocorpus de la norma lingüística culta de las principales ciudades del mundo bispánico (MC-NLCH), Corpus de conversaciones coloquiales (Val.Es.Co), Corpus Oral de Lenguaje Adolescente de Madrid (COLAm). De estas bases de datos extraemos las combinaciones más frecuentes en las que entra el marcador anda, vamos, vaya o venga; esto es, las que son iguales o superiores al umbral de frecuencia de cinco (ver 3) STET, lo cual resulta en un corpus de 589 ejemplos (tabla 1).

Para determinar la distinción entre combinaciones casuales y combinaciones que demuestran cierto grado de "atracción" entre sus constituyentes, aplicamos, en lo que sigue, una medida de asociación que se basa en la frecuencia relativa de la combinación y de sus constituyentes (sección 3). Asimismo, averiguamos, mediante un análisis cualitativo, si las combinaciones que más atracción demuestran desarrollan un valor holístico (sección 4).

\section{Metodología}

Estudios anteriores (Cuenca/Marín; Dostie 2013) se basan en las frecuencias observadas o absolutas de las combinaciones para seleccionar las combinaciones más frecuentes. Estas frecuencias absolutas de las combinaciones, sin embargo, no toman en cuenta las frecuencias de sus constituyentes y, por lo tanto, no informan sobre el grado de atracción entre estos constituyentes (Evert 1229). Consideremos, a título ilustrativo, las combinaciones y entonces y pues vale en el corpus de Val.Es.Co. La frecuencia absoluta de y entonces es 28 , mientras que pues vale ocurre no más de dos veces en el corpus. No obstante, los constituyentes de la combinación más frecuente ( $y$ y entonces) son ellos mismos marcadores muy frecuentes: $y$ y entonces ocurren, respectivamente, 3180 y 162 veces. En cambio, los constituyentes de la combinación pues vale son menos frecuentes con 543 ocurrencias de pues y 45 ocurrencias del marcador vale. Incluso cuando se reorganizan las palabras del corpus en un orden arbitrario, cabe esperar una fre- 
cuencia más alta de la combinación y entonces que de la combinación pues vale. Por ende, con base en su frecuencia absoluta es imposible pronunciarse sobre la casualidad o no casualidad de la recurrencia de una combinación. A ese respecto, señala Evert (1217) que "an additional measure of attraction strength is therefore needed in order to identify «true collocations» among the recurrent word pairs, or to distinguish between «strong» and «weak» collocations".

Si bien el método estadístico que propone Evert se aplica en su estudio a colocaciones léxicas (del tipo old man, cope with, radio emission, etc.), es igualmente aplicable, a nuestro juicio, a colocaciones de unidades pragmáticas. La medida de asociación propuesta por Evert calcula, con base en las frecuencias relativas, las fuerzas de asociación entre los constituyentes de una combinación. Las fuerzas de asociación permiten (a) distinguir entre las 'verdaderas' combinaciones y las combinaciones casuales y (b) redactar una lista de las combinaciones según la fuerza de asociación entre sus constituyentes.

La medida de asociación que aplicamos en el presente estudio para la combinación de dos marcadores es el cálculo de la información mutua puntual (IMP). La IMP es una medida de efecto del tamaño que calcula la correlación entre dos eventos $x$ y $y$. El cálculo interpreta la frecuencia observada de la combinación (O) en función de la frecuencia esperada (E) en una escala algorítmica: $\mathrm{IMP}=\log 2(\mathrm{O} / \mathrm{E})\left(\right.$ Evert 1230). ${ }^{3}$ Este cálculo genera para cada combinación un valor de IMP, esto es, una fuerza de asociación; un valor de IMP de 0 significa que la combinación ocurre con la frecuencia esperada $(\mathrm{O}=\mathrm{E})$ y que la recurrencia de la combinación de los dos marcadores es, por tanto, casual (lo cual es la hipótesis nula de la independencia). Cuanto mayor la IMP, menor será la casualidad de que se combinen los marcadores. Finalmente, un valor de IMP negativo indica que una combinación es menos frecuente que esperada y constituye, por tanto, evidencia de una anti-colocación (Evert 1231).

Ahora bien, para el cálculo de la IMP debe conocerse la frecuencia observada de la combinación, la cual se recupera fácilmente del corpus, y la frecuencia esperada que se calcula aplicando la fórmula $\mathrm{E}=\mathrm{f} 1 \mathrm{xf} 2 / \mathrm{N}$ en la que $\mathrm{f} 1$ y f2 son, respectivamente, las frecuencias del primer y del segundo marcador, y $\mathrm{N}$ es el tamaño del corpus entero. Por consiguiente, también es necesario recuperar las frecuencias de cada uno de los marcadores en el corpus.

3. La manera más intuitiva de calcular la medida de asociación es la ratio O/E, pero esta ratio puede ser extremamente alta en muestras muy grandes (porque $\mathrm{E}=1$ para algunas combinaciones). Por eso, es aconsejable calcular la asociación en una escala logarítmica en base 2 (log2) (Evert). 
Primero, presentamos las frecuencias absolutas de las combinaciones más frecuentes con anda, vamos, vaya y venga en nuestro corpus:

\begin{tabular}{|c|c|c|c|c|c|c|c|}
\hline ANDA & \# & VAMOS & \# & VAYA & \# & VENGA & \# \\
\hline pues anda & 11 & pero vamos & 299 & pues vaya & 15 & pues venga & 28 \\
\hline \multirow{2}{*}{$\begin{array}{l}\text { venga anda/ } \\
\text { anda venga }\end{array}$} & \multirow[t]{2}{*}{11} & y vamos & 49 & vaya hombre & 8 & venga venga & 23 \\
\hline & & porque vamos & 16 & \multirow[t]{8}{*}{ vaya vaya } & \multirow[t]{8}{*}{5} & bueno venga & 19 \\
\hline anda anda & 7 & venga vamos/ & 16 & & & a ver venga/ & 14 \\
\hline \multirow{6}{*}{$\begin{array}{l}\text { vamos anda/ } \\
\text { anda vamos }\end{array}$} & \multirow[t]{6}{*}{5} & vamos venga & & & & venga a ver & \\
\hline & & bueno vamos & 9 & & & vale venga/ & 11 \\
\hline & & vamos vamos & 8 & & & venga vale & \\
\hline & & \multirow[t]{3}{*}{ pues vamos } & \multirow[t]{3}{*}{7} & & & venga hombre & 10 \\
\hline & & & & & & $\begin{array}{l}\text { venga va/ } \\
\text { va venga }\end{array}$ & 10 \\
\hline & & & & & & y venga & 8 \\
\hline Total & 34 & Total & 404 & Total & 28 & Total & 123 \\
\hline
\end{tabular}

Tabla 1. Frecuencias absolutas de las combinaciones

Cabe añadir que se respeta un umbral de frecuencia absoluta de cinco $(f=5)$ para contrarrestar el sesgo de frecuencia baja, ya que la IMP tiende a asignar valores de asociación muy altos a combinaciones pocos frecuentes, sobre todo con datos de corpus extensos. Por eso, solo se consideran las combinaciones con una frecuencia de cinco o más.

Una segunda observación concierne a las combinaciones de anda, vamos, vaya y venga con una conjunción o un conector, a saber, pero, pues, porque y $y$. Según comenta Pons Bordería (157) "la simple adyacencia entre dos unidades no es garantía de la pertenencia al mismo constituyente", es decir, la estructura interna de vamos pero y de pero vamos es muy distinta. En el primer caso los dos constituyentes pertenecen a unidades estructurales distintas ([Tampoco es para tanto, vamos.] [Pero, aun así hay que tener cuidado.]), mientras que en el segundo caso el conector pero y el marcador modal vamos forman parte de la misma unidad estructural (Hay alguna excepción, [pero vamos], en general, no suele ser asî́. De esta manera, las combinaciones de uno de los marcadores objeto de estudio con un conector (por este orden) no son sino marcadores adyacentes de dos funciones distintas, por lo que las consideramos yuxtaposiciones meramente casuales.

Finalmente, no están incluidas las reduplicaciones (anda anda, vamos vamos, etc.) en el cálculo de la información mutua puntual, ya que no se pueden 
calcular sus fuerzas de asociación debido a la interdependencia de las constataciones de sus constituyentes. Por eso, recurrimos a su frecuencia absoluta y las tratamos como un tipo particular de combinación (4.2).

Además de las frecuencias observadas de las combinaciones, se necesita calcular la frecuencia esperada de cada combinación (tabla 4), lo cual se realiza con base en las frecuencias de sus constituyentes (tabla 2) y en el número de palabras del corpus entero (tabla 3):

\begin{tabular}{|l|r|}
\hline \multicolumn{1}{|c|}{ MARCADOR } & \multicolumn{1}{c|}{$\#$} \\
\hline a ver (marc.) & 1002 \\
\hline anda & 266 \\
\hline bueno (marc.) & 7219 \\
\hline hombre (marc.) & 1477 \\
\hline pero & 15642 \\
\hline porque & 9687 \\
\hline pues & (12 292 \\
\hline va (marc.) & 78 \\
\hline vale (marc.) & 1625 \\
\hline vamos & 1220 \\
\hline vaya & 108 \\
\hline venga & 624 \\
\hline$y$ & 40941 \\
\hline
\end{tabular}

Tabla 2. Frecuencias absolutas de los constituyentes ${ }^{5}$

\begin{tabular}{|l|r|}
\hline \multicolumn{1}{|c|}{ CORPUS } & \multicolumn{1}{c|}{$\#$} \\
\hline Val.ES.CO & 55183 \\
\hline COLAm & 175000 \\
\hline CORLEC & 1100000 \\
\hline C-ORAL-ROM & 300000 \\
\hline MC-NLCH & 110850 \\
\hline Total & 1741033 \\
\hline
\end{tabular}

Tabla 3. Número de palabras en el corpus

4. Hay que señalar que el marcador pues puede realizarse y transcribirse también como pos, pus o pueh.

5. Para las formas que pueden emplearse también como adjetivo, verbo o sustantivo (a ver, bueno, hombre, va y vale), solo se recogen las frecuencias de las formas usadas como marcadores del discurso (de ahí la abreviatura marc. en la tabla 2). 
Consideremos, a título ilustrativo, la combinación y vamos, cuyos miembros ocurren respectivamente 40941 (f1) y 1220 (f2) veces en un corpus con 1741033 palabras (N). La frecuencia esperada (E) de una combinación de los dos marcadores es $40941 \times 1220 / 1741033=28,69$. La frecuencia esperada (E) de $y$ vamos es, por lo tanto, sustancialmente más baja que la frecuencia observada ( $\mathrm{O}=49)$, lo cual es ya un indicio de su carácter colocacional (Evert). De esa manera se calculan las frecuencias esperadas de todas las combinaciones que se recogen en la tabla 4:

\begin{tabular}{|l|r|}
\hline \multicolumn{1}{|c|}{ ComBInACIÓN } & E=f1xf2/N \\
\hline pues anda & 1,88 \\
\hline venga anda/ anda venga & 0,10 \\
\hline vamos anda/ anda vamos & 0,19 \\
\hline pero vamos & 10,96 \\
\hline y vamos & 28,69 \\
\hline porque vamos & 6,79 \\
\hline venga vamos/vamos venga & 0,44 \\
\hline bueno vamos & 5,06 \\
\hline pues vamos & 8,61 \\
\hline pues vaya & 0,76 \\
\hline vaya hombre & 0,09 \\
\hline pues venga & 4,41 \\
\hline bueno venga & 2,59 \\
\hline a ver venga/ venga a ver & 0,36 \\
\hline vale venga/ venga vale & 0,58 \\
\hline venga hombre & 0,53 \\
\hline venga va/ va venga & 0,03 \\
\hline y venga & 14,67 \\
\hline
\end{tabular}

Tabla 4. Frecuencias esperadas de las combinaciones

Con base en estos datos cuantitativos se calcula el valor de IMP de cada una de las combinaciones. Así, por ejemplo, la fuerza de asociación de la combinación $y$ vamos es $\log 2(49 / 28,69)=0,77$, lo cual apunta a que $y$ vamos es efectivamente más frecuente de lo esperado y que hay, por tanto, cierta atracción entre sus dos constituyentes (aunque sea débil). La tabla 4 recoge todos los valores de IMP con las combinaciones con mayor fuerza de atracción encabezando la lista: 


\begin{tabular}{|l|c|}
\hline \multicolumn{1}{|c|}{ Combinación } & $\begin{array}{c}\text { IMP } \\
\text { Iog2(O/E) }\end{array}$ \\
\hline venga va/va venga & 8,48 \\
\hline venga anda/ anda venga & 6,85 \\
\hline vaya hombre & 6,45 \\
\hline a ver venga/ venga a ver & 5,28 \\
\hline venga vamos/vamos venga & 5,19 \\
\hline pero vamos & 4,77 \\
\hline vamos anda/ anda vamos & 4,75 \\
\hline pues vaya & 4,30 \\
\hline venga hombre & 4,24 \\
\hline vale venga/venga vale & 4,24 \\
\hline bueno venga & 2,88 \\
\hline pues venga & 2,67 \\
\hline pues anda & 2,55 \\
\hline porque vamos & 1,24 \\
\hline bueno vamos & 0,83 \\
\hline y vamos & 0,77 \\
\hline pues vamos & $-0,30$ \\
\hline y venga & $-0,88$ \\
\hline
\end{tabular}

Tabla 5. Valores de IMP de las combinaciones

La IMP de las combinaciones arroja nueva luz sobre la atracción entre sus constituyentes. Así, por ejemplo, a pesar de ser muy poco frecuente en cifras absolutas la combinación vaya hombre $(\mathrm{O}=8)$ resulta que vaya y bombre se combinan más frecuentemente de lo esperado en nuestro corpus. En cambio, los constituyentes de la combinación y venga, que tiene la misma frecuencia observada que la antedicha $(\mathrm{O}=8)$, parecen "repelerse" antes que atraerse, ya que la combinación tiene un valor de IMP $<0$. Otra anti-colocación es pues vamos con un valor de IMP de -0,30. Este método cuantitativo permite distinguir las combinaciones que muestran una atracción fuerte entre sus constituyentes de las combinaciones casuales. Se espera, por lo tanto, una cierta afinidad pragmática entre las coocurrencias no casuales.

En lo que sigue, abordamos tres cuestiones más concretas y cualitativas concernientes a las combinaciones. En primer lugar, discernimos diferentes tipos de combinaciones con base en sus estructuras internas (4.1); luego, ahondamos en el porqué de la atracción de los constituyentes (4.2); finalmente, verificamos la hipótesis del valor sumativo de las combinaciones y nos preguntamos, pues, en qué medida las combinaciones están "rutinizadas" (4.2 y 4.3). 


\section{RESULTADOS Y DisCUSIÓN}

\subsection{Estructura interna}

Cuenca y Marín (904) disciernen, entre los constituyentes de las combinaciones, cuatro tipos de marcadores, a saber, las conjunciones (y, pero, porque), los conectores parentéticos (entonces, pues), los conectores pragmáticos (claro, hombre, bueno) y las interjecciones (exacto, uy). Según el modelo de los marcadores propuesto por Cuenca $(2013,210)$ las conjunciones, los conectores parentéticos y los conectores pragmáticos son prototípicamente marcadores discursivos (MDi), ya que son "two position operators", esto es, concretan el contenido de dos enunciados. Las interjecciones, en cambio, son marcadores modales (MM) porque son "one position operators": modifican la intención de un único enunciado.

Efectivamente, en nuestro corpus discernimos, por un lado, las combinaciones de un marcador de un verbo de movimiento y un marcador discursivo (a partir de ahora $[\mathrm{MDi}+\mathrm{M}]$ ) y, por otro lado, las combinaciones de un marcador de un verbo de movimiento con un marcador modal (a partir de ahora $[\mathrm{MM}+\mathrm{M}])$. El primer grupo incluye a las combinaciones en las que entran $y$, pero, pues, bueno y porque. Los marcadores modales que se combinan con los marcadores objeto de estudio son va, hombre, a ver y vale. Dentro del grupo $[M M+M]$ encontramos también las combinaciones de los marcadores de los verbos de movimiento entre sí (venga anda, venga vamos, vamos anda). ${ }^{6}$

A estos dos tipos estructurales de combinaciones se añade un tercer tipo, a saber, la reduplicación de un mismo marcador (anda anda). En lo que sigue, averiguamos cómo estos tres tipos se distinguen en cuanto a sus intenciones comunicativas y nos preguntamos por qué se atraen los dos constituyentes dentro de una combinación.

\subsection{Integración funcional}

El análisis semántico-pragmático de las combinaciones expone varios grados de integración funcional según los tipos estructurales de las combinaciones. Concretamente, los tres tipos se sitúan en un continuo en el que las reduplicaciones demuestran una integración funcional completa, mientras que las combinaciones del tipo $[\mathrm{MDi}+\mathrm{M}]$ tienen el menor grado de integración funcional.

6. Como veremos a continuación, dentro del tipo $[M M+M]$ el orden interno de los constituyentes es libre. 


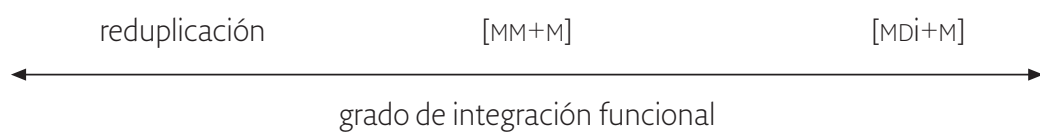

Figura 1. Continuo del grado de integración funcional

En el corpus hemos encontrado 40 ejemplos de reduplicaciones del mismo marcador con el objetivo de realzar su valor apelativo (18) (19) o expresivo (20) (21):?

18) MALCE2J04: es que mirar os cuento. a mí me gusta <navn>Juanma $</$ navn $>$

MALCE2J01: 2 [espera vamos vamos] (COLAm)

19) $<$ H2> Buenos días, José Luis. Un día más aquí con... con todos ustedes para contarles... bueno pues un montón de suculentas noticias $<$ simultáneo > <ininteligible>

$<$ H1 $>$ Venga, venga. Estamos ansiosos. (CORLEC)

20) $<\mathrm{H} 2>$ ¡Ah! ¡Ah, tío, me estabas asustando, yo ya había llama $<$ (d) $>$ o a unos colegas que andan apura $<(\mathrm{d})>$ os de pelas y no saben qué hacer este viernes y les he dicho: "Oye, dan un concierto de puta madre, veniros conmigo"

$<$ H1 $>$ Es gratis y además... < fático $=$ duda $>$ os vamos a invitar a una cervecita... Aquí eh... "Canela en Rama" se va a tirar el rollo, $<$ simultáneo> una vez en la vida...

$<$ H2> ¡Anda, anda! </simultáneo $>$ (CORLEC)

21) $<\mathrm{H} 3>$ A mí me nombraron presidente y administrador hace... 6 años. Y... al mes ya lo había deja $<$ (d) $>$ o. ¡Era horrible! ¡Horrible! $<\mathrm{H} 2>$ ¡Vaya, vaya! (CORLEC)

La reduplicación de un marcador tiene, según Dostie (2007, 57), un doble efecto: se realiza un acto ilocutivo de insistencia y, al mismo tiempo, el hablante expresa una implicación más marcada en comparación con el uso no reduplicado. El efecto de insistencia se explica por el principio de cantidad, que postula que más forma corresponde a más significado (Haiman). En este caso,

7. En el corpus hay también tres ejemplos de vamos reduplicado con función metadiscursiva. No obstante, el objetivo no es insistir en su valor, sino que señala vacilación por parte del hablante: "C: $\$$ y yo digo ¡ostras! cinco mil pesetas en- en na(da)/ porque esto / vamos... vamos / porque es que era un señor pero muy serio oye? y que- que- que [te daba hasta miedo ¿eh?]" (Val.Es.Co). 
la abundancia de material lingüístico (la reduplicación) procura la intensificación (Landone 334).

Cabe subrayar que solo los marcadores que se usan como interjecciones, esto es, que tienen valor apelativo o expresivo y que pueden, por tanto, ser equivalentes no-prototípicos de enunciados, se reduplican con el efecto de insistencia, lo cual confirma la hipótesis de Dostie $(2007,58)$ de que "la [réduplication pragmatique] des [marqueurs discursifs] concerne souvent les sens où ces unités réalisent un acte illocutoire et où elles ont donc le status de motsprases (ex.: OK OK, voyons voyons, tiens tiens, bien bien, mais non spontanément ??puis puis...)".

En este sentido las combinaciones del tipo $[M M+M]$ se parecen a las reduplicaciones en cuanto a su valor pragmático-semántico: los dos constituyentes sirven a una intención comunicativa apelativa o expresiva muy afín. Consideremos los ejemplos siguientes:

22) $<\mathrm{H} 2>$ Oye ¿no te quieres ir hoy a dormir a casa de Lolita? $<$ H4> No. Hoy no. Está durmiendo Conchi y no hay sitio. $<$ H5 > Venga, a ver, cuenta. (CORLEC)

23) *TRI: $[<]<$ si se> portara como se tiene que portar / todavía dices / venga / vale / pues te / ayudo / y tal //\$ pero si es [/] pero si es que ha estado de viviendo (sic) de mamá toda la vida (C-ORAL-ROM)

En el ejemplo (22) la combinación venga a ver tiene función apelativa, ya que tanto venga como a ver pueden emplearse para apelar al interlocutor (A ver, Merche, déjame pasar) (Fuentes Rodríguez 2009; Santos Río; Tanghe). La combinación venga vale en el ejemplo (23) se usa para expresar un asentimiento o la aceptación de una propuesta. Es bien sabido que vale se usa siempre con valor de acuerdo o de conformidad (Fuentes Rodríguez 2009; Padilla García; Santos Río), contrariamente al otro constituyente de la combinación venga que es polifuncional. Por ello, parece que el marcador pragmáticamente más especializado (vale) anula en estos contextos la posible vaguedad del marcador polifuncional (venga).

Aun así, no necesariamente se combina un marcador con valor especializado con otro más polifuncional; también es frecuente la combinación de dos marcadores polifuncionales, más concretamente cuando coocurren dos marcadores objeto de estudio:

24) *PAC: dice hhh / bueno ya te \&con $+\$$ digo venga anda / súbete luego si quieres a tomar café / y no sé qué //\$ (C-ORAL-ROM) 
25) MALCC2G02: porque a lo mejor

$<\mathrm{R}>$ porque me iba a

MALCC 2G02: sentar así en una silla

y no iba hacer nada

vamos andaaa $</ R>$

MALCC2G01: porque a lo mejor

tú ves una faceta de ti

que dices

ala que guapo (COLAm)

En el ejemplo (24) la combinación venga anda tiene valor apelativo, es decir, los dos constituyentes de la combinación tienen valor apelativo mientras que en el ejemplo (25) los dos constituyentes tienen valor expresivo (de incredulidad o asombro). En estos ejemplos es el contexto el que desambigua la función de los dos marcadores y, por lo tanto, de la combinación.

$\mathrm{Al}$ igual que la reduplicación de un marcador, los valores de los dos constituyentes de $[M M+M]$ se solapan en gran medida, ya que los dos sirven a intenciones comunicativas modales muy afines. En consecuencia, igual que las reduplicaciones, las combinaciones del tipo $[\mathrm{MM}+\mathrm{M}]$ procuran insistir en el acto ilocutivo que expresan añadiendo más material lingüístico. Prueba del valor afín de los dos constituyentes es la libertad en la estructura interna de este tipo de combinaciones. Así, la alternancia entre venga vale y vale venga o entre anda venga y venga anda parece ser libre, esto es, no implica un cambio en la intención comunicativa:

26) MAESB2J01: solo somos tres tal

MAESB2G03: vale venga pasar (COLAm)

27) MALCC2G03: nada que bebes no\venga da $\mathrm{xxx}$

MALCC2G05: anda venga bebe <sonido de aspiración/> (COLAm)

En el ejemplo (26) la combinación vale venga tiene valor de aceptación de una propuesta, igual que venga vale en el ejemplo (23). En el ejemplo (27) la combinación anda venga cumple una función apelativa tal y como la combinación venga anda en el ejemplo (24). De la intención comunicativa afín de los constituyentes y de la correspondiente libertad interna de las combinaciones se concluye que los constituyentes pertenecen a la misma unidad discursiva.

Como excepción dentro de la categoría de $[M M+M]$ destacamos las combinaciones con hombre, cuya estructura interna es fija, ya que hombre siempre 
se pospone. Identificamos dos combinaciones con hombre con un valor de IMP positivo, a saber, venga hombre $(\mathrm{IMP}=4,24)$ y vaya hombre $(\mathrm{IMP}=6,45)$ :

28) <H6> Y vamos a ir, en esta pantalla a ver... < vacilación> ¡vaya hombre, he perdido el... < vacilación> el cartón! ¿Dónde lo tengo? Aquí. (CORLEC)

29) <H3> Pensar que tengo que salir ahora con mi coche, a ese lío.

Venga, hombre, yo me quedo aquí. Yo les espero, $<$ H1> Huy, ¡qué horror! (CORLEC)

Como es bien sabido, la partícula hombre tiene un estatuto ambiguo: según describen Briz Gómez y Villalba, hombre se usa como partícula apelativa, reforzando el acuerdo o atenuando el desacuerdo con el interlocutor, o como reafirmador de lo hecho o dicho por el hablante mismo. En otras palabras, se usa como vocativo fático o como marcador modal, respectivamente. A este respecto, no es de extrañar que hombre prefiera posponerse al marcador vaya o venga, ya que un vocativo en posición final de un enunciado tiene valor reforzador o atenuador del acto comunicativo (Bañón; García Dini; Cuenca 2004). Así, en el ejemplo (28) el hablante no apela a ningún interlocutor con el uso de hombre, sino que refuerza el acto comunicativo de asombro y desagrado expresado por vaya.

En suma, cuando hay una zona de solapamiento pragmático completa (en el caso de marcadores reiterados) o parcial (en el caso de las combinaciones del tipo $[M M+M])$, se logra un efecto de insistencia en el acto ilocutivo. Como los dos constituyentes tienen la misma intención comunicativa, forman una unidad discursiva y tienen una estructura interna libre, sin que un marcador tenga alcance sobre otro.

Con respecto al tipo de combinación $[\mathrm{MDi}+\mathrm{M}]$ constatamos que la integración funcional es más bien limitada. Aun así, los valores de IMP positivos de combinaciones como y vamos, pero vamos, pues venga, etc. demuestran que existe cierta atracción entre los dos constituyentes y que, por tanto, las combinaciones no son aleatorias. Consideremos los ejemplos siguientes:

30) E: yo sí / liberaal- soy conservadora enn-/ pues en lo que interesa como to'l mundo... pero vamos no soy nada liberal? lo contrario (Val.Es.Co)

31) Inf.- Pues esto es muy difícil. Desde luego, los jóvenes de hoy se encuentran con una civilización mil veces más abierta que la... que la... que la que hemos encontrado nosotros, porque, vamos, en mis 
tiempos, los padres eran sagrados y lo que decían no se discutía nada; (MC-NLCH)

32) joder, casi hasta se ha enfada $<$ (d) $>$ o también el pobre, claro, luego he visto que algún defectito tenía, ¿no? "Pues yo tengo un amigo que trabaja el vidrio" Pues anda, un amigo trabaja el vidrio, ya, pero para hacer las botellas hace falta almuflas, hace falta tener una fábrica a la espalda, pero uste $<$ (d) $>$ no comprende, mira, si esto es, mira, una botella de suero, (CORLEC)

En el ejemplo (30), el marcador pero conecta el enunciado que sigue con el enunciado que precede; el lazo proposicional entre los dos enunciados es adversativo restrictivo. Al mismo tiempo, pero señala la importancia del segundo miembro para sacar las conclusiones pertinentes (Santos Río). El marcador vamos, a su vez, predica meramente sobre el enunciado que sigue y subraya su fuerza argumentativa. De esta manera, los valores de los dos marcadores se solapan parcialmente: ambos marcadores indican la importancia en un nivel argumentativo del contenido del enunciado que sigue (no soy nada liberal). Como veremos a continuación (4.3), la combinación pero vamos se emplea con valores particulares que se relacionan con este valor de recalcar la fuerza argumentativa del enunciado que sigue.

Los dos constituyentes de la combinación porque vamos en el ejemplo (31), en cambio, no demuestran solapamiento funcional: la conjunción porque introduce una causa y aclara la información que precede, mientras que vamos subraya la fuerza argumentativa del enunciado que sigue. La fuerza de asociación entre porque y vamos se explica por la índole subjetiva del enunciado introducido en el que el hablante suele expresar su punto de vista u opinión. De esta manera, porque indica que en lo que sigue se aclara lo que precede y vamos subraya la importancia para el hablante de esta aclaración. Los valores de los dos constituyentes son, por tanto, meramente complementarios.

Igualmente, en la combinación pues anda del ejemplo (32) los valores de los dos constituyentes demuestran afinidad pragmática sin solaparse. El conector pragmático pues introduce un enunciado reactivo (Fuentes Rodríguez 2009) y el marcador modal anda constituye esta reacción, ya que tiene valor expresivo de desacuerdo o rechazo.

Todo lo anterior explica por qué consideramos las combinaciones del tipo $[\mathrm{MDi}+\mathrm{M}]$ como un tipo particular. Como el marcador discursivo concreta el contenido de dos enunciados, tiene el alcance sobre el enunciado que precede y el enunciado que sigue. Por consiguiente, el marcador discursivo se an- 
tepone siempre al marcador de verbo de movimiento, cuyo alcance se limita al enunciado que sigue. Los dos constituyentes no pertenecen, pues, a la misma unidad discursiva ya que operan en dos niveles estructurales distintos. Por eso, la estructura interna de este tipo de combinaciones es fija: el marcador discursivo precede necesariamente al otro marcador, que suele tener función más bien modal.

Resumiendo, los tipos de marcadores que se combinan (marcador modal o marcadores discursivos) tienen sus repercusiones en la intención comunicativa y en la integración funcional de la combinación. Así, las reduplicaciones y las combinaciones del tipo $[\mathrm{MM}+\mathrm{M}]$ tienen función apelativa o expresiva y los valores de los constituyentes se solapan completamente o parcialmente, logrando así una insistencia en el acto ilocutivo. Además, en las combinaciones del tipo $[\mathrm{MM}+\mathrm{M}]$, el orden de sus constituyentes es libre, mientras que en las combinaciones del tipo [MDi+M] el orden es fijo, porque los constituyentes operan en niveles estructurales distintos. Sus constituyentes se combinan tan frecuentemente no para lograr una insistencia, sino porque sus valores resultan ser sumamente complementarios.

Retomando la pregunta de investigación inicial del valor o bien sumativo o bien holístico de las combinaciones, concluimos que cada uno de los constituyentes mantiene su valor individual al entrar en una combinación, incluso los constituyentes con una fuerza de atracción fuerte (venga va, venga anda). Las combinaciones que muestran un alto grado de integración funcional no necesariamente han desarrollado un valor pragmático distinto de la suma de los valores de sus constituyentes, es decir, los valores de los constituyentes no se amalgaman en un valor holístico distinto de la suma de sus partes; sino que, por tener valores muy afines o complementarios, algunos marcadores se atraen y se combinan más frecuentemente que otros. En otras palabras, los marcadores combinados colaboran para lograr cierto objetivo comunicativo, como señala Linell (322): "Linguistic resources are typically co-selected in discourse. Such resources will often mutually influence what aspects of their meaning potentials are reinforced in the situated utterance".

Aun así, según el principio de "entrenchment" las combinaciones más frecuentes (en cifras absolutas) deberían estar "rutinizadas" en cierta medida (Langacker 59), lo cual implica una fijación de rasgos formales y funcionales. Efectivamente, una de las combinaciones demuestra indicios formales y funcionales de estar fijada como unidad discursiva. Entramos en detalle, en lo que sigue, en el cómo y el porqué del comportamiento particular de pero vamos. 


\subsection{La rutinización de pero vamos}

La combinación pero vamos demuestra una IMP positiva y relativamente alta $(4,77)$, es decir, sus constituyentes pero y vamos se combinan más frecuentemente de lo esperado, lo cual es prueba de una atracción entre los valores de los dos constituyentes. Por otro lado, pero vamos tiene una frecuencia de caso (token frequency) muy elevada para con las demás combinaciones (299 que es más de 6 veces más frecuente que la segunda combinación más frecuente: $y$ vamos, 49) (tabla 1). Una alta frecuencia de caso ha sido relacionada en la literatura con la rutinización de signos lingüísticos (Lehmann; Detges/Waltereit). Si bien los autores no se pronuncian sobre la frecuencia como un impulso para la rutinización o, en cambio, como una consecuencia de la rutinización, no cabe duda de que, en un nivel sincrónico, una alta frecuencia de caso va de la mano con una rutinización de un signo lingüístico. ${ }^{8}$

En cuanto a los marcadores del discurso, proponen Detges y Waltereit que "from a strictly synchronic point of view, not only grammatical items in the narrow sense, but also modal particles and discourse markers represent (different types of) procedural routines". Según los autores, los marcadores del discurso son un tipo de rutinas de procesamiento de las que disponen los hablantes para gestionar las actividades conversacionales y para solucionar varios tipos de problemas comunicativos. Ahora bien, dada la alta frecuencia de pero vamos, proponemos averiguar si la combinación se emplea como una rutina de procesamiento propia que gestiona actividades conversacionales particulares y distintas de la suma de los valores de pero y vamos.

Antes que nada, cabe señalar la flexibilidad pragmática de la combinación: distinguimos por lo menos cuatro actividades conversacionales que puede cumplir pero vamos. En primer lugar, como ya hemos señalado (4.2), la combinación pero vamos indica la importancia en un nivel argumentativo del enunciado que sigue:

33) *INT: $[<]<$ sí> //\$ mejor llamarla / no sea que se la pase / y estar un poquito encima de ella $\ldots \$$

${ }^{*}$ GAR: vale //\$

*INT: pero vamos / que esa [/] esa va a ser la más fácil / yo creo //\$ o sea que podemos empezar por ahí <para> [/ $\$$ (C-ORAL-ROM)

8. Así, por ejemplo, afirman Detges y Waltereit que "high frequency, in turn, leads to routinization of these items" $y$, al mismo tiempo, postulan que "routinization makes a linguistic sign more frequent". 
De esta manera, pero vamos recalca la fuerza argumentativa del enunciado que sigue. Los otros tres usos de la combinación se relacionan con este valor de refuerzo argumentativo. Primero, pero vamos resalta la importancia del enunciado posterior que especifica o matiza lo que antecede:

34) me dieron útil condicional, porque era estrecho de pecho; total, aprobé, pero, vamos condicionalmente (MC-NLCH)

Segundo, centrando la importancia informativa en el enunciado que sigue, se resta importancia a lo que precede. De ahí que se emplee pero vamos también para dar por terminado un tema o concluir una digresión:

35) Enc.- Vamos a ver, Emilia, tus padres, ¿¿dónde nacieron?

Inf.- Pues verás, mi padre nació aquí en Sevilla. Mi madre, no. Mi madre es... ¿Cómo se llama esto?... Yo no me acuerdo... De un pueblo... De Rociana, eso. Pero vamos, con un año creo que se vino a Sevilla, o sea, que ella es de Sevilla prácticamente. (MC-NLCH)

36) Inf.- Pues ahí también iba mucho la gente a tomar el té. Ahí se reunía la gente así conocida, ¿verdad?, todos así íbamos a esos sitios, o a un sitio o a otro, pero vamos... Este de la Gran Vía ya no me acuerdo ahora cómo se llama [...]. (MC-NLCH)

En el ejemplo (35), el hablante recurre a la combinación pero vamos para dar por terminada una digresión y centrarse de nuevo en el argumento más pertinente de su discurso. En el ejemplo (36) la combinación indica que el hablante da por terminado el tema del discurso. En este ejemplo la combinación se encuentra en posición final de enunciado dejando libre, así, la continuación del discurso. La combinación pero vamos en esta posición funciona, por lo tanto, como un tipo de marqueur de balisage, señalando "la fin d'une étape dans una intervention" (Dostie 2004, 48). Este uso se relaciona directamente con el uso de pero vamos para ceder el turno ("turn-yielding") (Duncan):

37) MALCC2J03: ssii no o sea quee que su primo le había dicho que no lo sabía si iba ir y tal entonces que solo no le apetecía ir solo para enseñar el pibe de la camiseta y eso y que si al final no iba pues quedaban todos pero ya por la noche a cenar y tal pero vamos

MALCC2J01: ah luego quedamos a hacer empanadillas para cenar si os apetece si os gustan (COLAm)

38) $<\mathrm{H} 7>\dot{\mathrm{T}} \mathrm{Te}$ acuerdas que nos comieron el saco y se nos salieron unos pocos? 
$<$ H6> Pero si na<(d)>a más había que ver las laderas que hay... pasa $<$ (d) $>$ o el cerca $<$ (d) $>$ o de piedra para allá había unas laderas... que están todas agujereadas que allí había conejos pero vamos... $<\mathrm{H} 2>$ ¿Y... y en las tardes, en las noches al escurecer? (CORLEC)

En este ejemplo con el uso de pero vamos el hablante cede su turno de habla ofreciendo la oportunidad al interlocutor de tomar el turno.

Resumiendo, el corpus demuestra que la combinación pero vamos se ha especializado en la gestión de varias actividades conversacionales, relacionadas todas con el valor de refuerzo argumentativo de los dos constituyentes pero y vamos. Constatamos, además, que pero vamos ha adoptado un uso cuyo valor no se deja describir por la suma de los valores de sus constituyentes, a saber, el uso de cesión de turno. Mientras que pero vamos suele operar en un nivel metadiscursivo cuando focaliza la información del enunciado que sigue o da por terminado un tema, la combinación opera en un nivel interaccional cuando se emplea para estructurar la alternancia de turnos. Proponemos, por tanto, que con la función de ceder el turno pero vamos se usa como un conjunto en el que el todo es mayor que la suma de sus partes.

Constatamos que este valor específico lleva aparejada una distribución y configuración prosódica específica. En primer lugar, pero vamos con valor de cesión de turno se encuentra siempre en posición final de la intervención:

39) *RAU: $[<]<$ vale $>/ / \$$ pues entonces ya está //\$ yo cuando [/] cuando sea / te llamo [/] te llamo a casa / o me paso // $\$$ ya sabes $<$ que $>+\$$ *MAN: $[<]<$ o si no $>$ me puedes llamar aquí //\$ por la mañana estoy aquí / pero vamos ...\$

*RAU: y cuál es el teléfono ?\$ este ?\$ (C-ORAL-ROM)

Esta posición final no es de extrañar, ya que, como afirman Beeching y Detges (11), la periferia derecha es la posición predilecta para gestionar la cesión de turno.

En segundo lugar, el análisis prosódico de los ejemplos de pero vamos en posición final de intervención de los cuales disponemos del audio corrobora que se emplea la combinación para indicar el fin de la intervención del hablante y para otorgar la posibilidad de tomar el turno al interlocutor, ya que el tonema es descendente. Consideremos el ejemplo siguiente con la curva melódica estilizada correspondiente:

40) MAORE2J01: 2 [o sea me dice mi amiga me dice mi amiga] MAORE2J04: 2 [ya yo lo sabía eh/ <navn>alicia</navn>] 
MAORE2J01: 1[oye el sulfero]

MAORE2J04: 1[yo sabía que estaba a por tí]

MAORE2J01: 2 [es que yo en la fiesta me dí (sic.) cuenta pero vamos] MAORE2J05: 2 [y desde cuando]

MAORE2J04: no yo me dí (sic.) cuenta antes (COLAm)

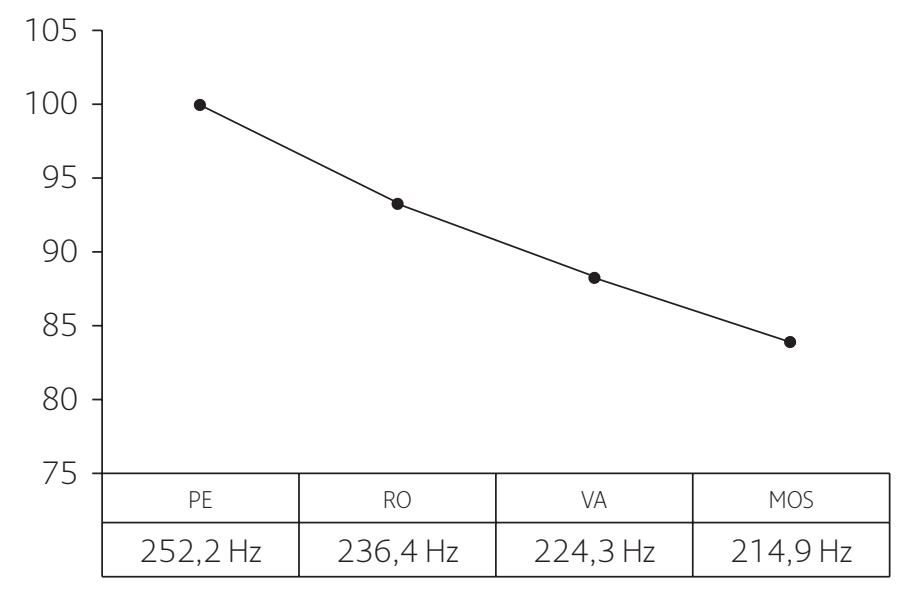

Figura 2. Curva melódica estilizada de pero vamos ${ }^{9}$

El ejemplo (40) y la correspondiente curva estilizada ilustran que el interlocutor no tiene la intención de continuar hablando: no es un acto suspendido, porque no se pronuncia con tonema ascendente o suspendido (Grupo Val.Es.Co 52). Al contrario, el tonema descendente de pero vamos señala el fin de la intervención del hablante.

De la combinación de reducción de composicionalidad al nivel semántico y el uso específico al nivel distribucional y prosódico concluimos que pero vamos es una micro-construcción con un emparejamiento formal-funcional. Con el valor de cesión de turno, la combinación conoce un alto grado de consolidación formal y funcional y está sedimentada (entrenched) como una unidad en la mente de los hablantes (Langacker 59). Tomando en cuenta el aspecto de frecuencia, no está excluido que otras combinaciones con una fuerza de asociación bastante fuerte y una frecuencia de caso relativamente alta (porque vamos, pues venga) vayan especializándose cada vez más a nivel formal y funcional.

9. Aplicamos el análisis melódico del habla propuesto por Cantero Serena y Font Rotchés para la estilización de la curva melódica. 


\section{Conclusiones}

El punto de partida del presente estudio fue la observación de la frecuente combinación de dos (o más) marcadores en el lenguaje hablado. Como sujeto de estudio optamos por los marcadores derivados de verbos de movimiento por su flexibilidad pragmático-semántica y la frecuente combinación de estos marcadores con otros (tanto modales como discursivos). En una primera fase propusimos un método cuantitativo que permite distinguir entre los marcadores que se combinan por casualidad y los que se combinan por mostrar una atracción mutua. De esta manera, identificamos un grupo de 16 combinaciones, dentro del cual distinguimos tres tipos de combinaciones, a saber, los marcadores objeto de estudio que se reduplican, los que se combinan con un marcador modal y los que se combinan con un marcador discursivo. Esta estructura interna resulta tener repercusión en la integración funcional de las combinaciones. Así, los valores de los constituyentes de las reduplicaciones y del tipo $[\mathrm{MM}+\mathrm{M}]$ se solapan completamente o parcialmente, lo cual procura una insistencia en su acto ilocutivo. Los constituyentes de las combinaciones del tipo $[\mathrm{MDi}+\mathrm{M}]$ se atraen porque sus valores son complementarios. De todos modos, las combinaciones estudiadas suelen tener un valor ante todo sumativo, esto es, su valor es el resultado de la suma de los valores de sus constituyentes.

Una combinación llamó la atención por su alta frecuencia de caso y su flexibilidad pragmático-semántica, lo cual nos hizo sospechar que pero vamos está más rutinizado que las demás combinaciones. Se constató, en efecto, que pero vamos muestra un uso específico, de cesión de turno, con una consolidación a nivel pragmático-semántico y formal (posición discursiva de final de intervención y curva melódica descendente).

A modo de conclusión, sería interesante examinar el camino histórico que ha seguido pero vamos para fijarse como micro-construcción y encontrar, de este modo, el contexto crítico (Diewald) que ha desencadenado el proceso de fijación formal y funcional de la combinación. Además, el presente estudio cubre solo un grupo de marcadores muy limitado (el de los derivados de verbos de movimiento). La investigación de un grupo más amplio de marcadores más diversos podría revelar más combinaciones fijadas como micro-construcciones. De la comparación y el estudio detenido, en un nivel sincrónico y diacrónico, de este tipo de micro-construcciones (sus estructuras internas, sus funciones discursivas, etc.) podría aprenderse más sobre las estrategias comunicativas a las que recurren los hablantes para solucionar problemas discursivos. 


\section{OBRAS CITADAS}

Bañón, Antonio Miguel. El vocativo: propuestas para su análisis lingüístico. Barcelona: Octaedro, 1993.

Beeching, Kate y Ulrich Detges. Discourse Functions at the Left and Right Periphery: Crosslinguistic Investigations of Language Use and Language Change. Leiden/Boston: Brill, 2014.

Briz Gómez, Antonio y Cristina Villalba. "Hombre". Diccionario de partículas discursivas. Coords. Antonio Briz, Salvador Pons y José Portolés. 2004. 6 de julio de 2016. <www.dpde.es>.

Briz Gómez, Antonio, Salvador Pons Bordería y José Portolés, coords. Diccionario de partículas discursivas del español (2004). 6 de julio de 2016. <www. dpde.es>.

C-ORAL-ROM: Emanuela Cresti y Massimo Moneglia. C-ORAL-ROM: Integrated Reference Corpora for Spoken Romance Languages. Amsterdam: Benjamins, 2005.

Cantero Serena, Francisco José y Dolors Font Rotchés. "Protocolo para el análisis melódico del habla". Estudios de Fonética Experimental 18 (2009): $17-32$.

Castillo Lluch, Mónica. "La formación de los marcadores discursivos vaya, venga, anda y vamos". Actas del VII congreso internacional de bistoria de la lengua española. Ed. Concepción Company Company. Madrid: Arco Libros, 2008. 1739-52.

COLAm: Annette Myre Jørgensen. Proyecto Cola: Corpus Oral de Lenguaje Adolescente de Madrid. 6 de julio de 2016. <http://www.colam.org/om_prosjespannol.html>.

CORLEC: Francisco Marcos Marín, dir. Corpus de Referencia de la Lengua Española Contemporánea. 1 de mayo de 2016. <http://www.lllf.uam.es/ESP/ Corlec.html>.

Cuenca, Maria Josep. "El receptor en el text: el vocatiu". Estudis Romànics 26 (2004): 39-63.

Cuenca, Maria Josep. "The fuzzy boundaries between discourse marking and modal marking". Discourse Markers and Modal Particles: Categorization and Description. Eds. Liesbeth Degand, Bert Cornillie y Paola Pietrandrea. Amsterdam/Philadelphia: John Benjamins, 2013. 191-216.

Cuenca, Maria Josep y Maria Josep Marín. "Co-occurence of discourse markers in Catalan and Spanish oral narrative". Fournal of Pragmatics 41 (2009): 899-914. 
Detges, Ulrich y Richard Waltereit. "Grammaticalization and Pragmaticalization". Manual of Grammatical Interfaces in Romance. Eds. Susann Fischer y Christoph Gabriel. Berlin: De Gruyter Mouton, 2016. 635-58.

Diewald, Gabriele. "Context Types in Grammaticalization as Constructions". Constructions. Special Volume 1. 6 de julio de 2016. <http://journals.linguisticsociety.org/elanguage/constructions/issue/view/17.html>.

Dostie, Gaétane. Pragmaticalisation et marqueurs discursifs: analyse sémantique et traitement lexicographique. Bruxelles: De Boeck Duculot, 2004.

Dostie, Gaétane. "La réduplication pragmatique des marqueurs discursifs: de là à là là". Langue française 2.154 (2007): 45-60.

Dostie, Gaétane. "Les associations de marqueurs discursifs - De la cooccurrence libre à la collocation”. Linguistik Online 62.5 (2013): s.p.

Duncan, Starkey. "Some Signals and Rules for Taking Turns in Conversation". Fournal of Personality and Social Psychology 23.2 (1972): 283-92.

Evert, Stefan. "Corpora and Collocations". Corpus Linguistics: An International Handbook. Eds. Anke Lüdeling y Merja Kytö. Berlin: Mouton De Gruyter, 2008. 1212-48.

Fuentes Rodríguez, Catalina. "Vamos: un conector coloquial de gran complejidad”. Marcadores del discurso: teoría y análisis. Eds. María Antonia Martín Zorraquino y Estrella Montolío Durán. Madrid: Arco Libros, 1998. 177-92.

Fuentes Rodríguez, Catalina. Diccionario de conectores y operadores del español. Madrid: Arco Libros, 2009.

García Dini, Encarnación. "Algo más sobre el vocativo". Atti del XVII convegno [Associacione Ispaniste Italiani]. Vol. 2. Roma: Bulzoni, 1998. 57-62.

Gras Manzano, Pedro, Fernando Polanco Martínez y Marisa Santiago Barriendos. "Forma, función y evolución del marcador conversacional venga en español". Actas del VI Congreso de Lingüística General, II: Las lenguas y su estructura (IIa). Coord. Pablo Cano López. Madrid: Arco Libros, 2004. 1621-36.

Grupo Val.Es.Co. "Las unidades del discurso oral: la propuesta Val.Es.Co de segmentación de la conversación (coloquial)". Estudios de Lingiuística del Español 35.1 (2014): 11-71.

Haiman, John. Iconicity in Syntax. Amsterdam/Philadelphia: John Benjamins, 1985.

Hummel, Martin. Polifuncionalidad, polisemia y estrategia retórica: los signos discursivos con base atributiva entre oralidad y escritura; acerca de esp. bueno, claro, total, realmente, etc. Berlin: De Gruyter, 2012. 
Landone, Elena. Los marcadores del discurso y la cortesía verbal en español. Bern/ New York: Peter Lang, 2009.

Langacker, Ronald W. Foundations of Cognitive Grammar, I: Theoretical prerequisites. Stanford (Calif.): Stanford UP, 1987.

Lehmann, Christian. Thoughts on Grammaticalization. München: Lincom Europa, 1995.

Linell, Per. Rethinking Language, Mind and World Dialogically: Interactional and Contextual Theories of Human Sense-Making. Charlotte, NC: Information Age Publishing, 2009.

MC-NLCH: José Antonio Samper Padilla. Macrocorpus de la norma lingüistica culta de las principales ciudades del mundo hispánico. Las Palmas de Gran Canaria: Universidad de Las Palmas de Gran Canaria, 1998.

Montolío Durán, Estrella. "La teoría de la relevancia y el estudio de los marcadores discursivos". Los marcadores del discurso: teoría y análisis. Eds. María Antonia Martín Zorraquino y Estrella Montolío Durán. Madrid: Arco Libros, 1998. 93-119.

Mosegaard Hansen, Maj-Britt. The Function of Discourse Particles: A Study With Special Reference to Spoken Standard French. Amsterdam/Philadelphia: John Benjamins, 1998.

Mosegaard Hansen, Maj-Britt. Particles at the Semantics/Pragmatics Interface: Synchronic and Diachronic Issues. A Study With Special Reference ot the French Phasal Adverbs. Oxford: Elsevier, 2008.

Octavio de Toledo y Huerta, Álvaro S. "¿Un viaje de ida y vuelta?: la gramaticalización de vaya como marcador y cuantificador". Anuari de filologia 23-24 (2001-2002): 47-71.

Padilla García, Xose A. "Vale". Diccionario de partículas discursivas. Coords. Antonio Briz, Salvador Pons y José Portolés. 2004. 6 de julio de 2016. $<$ www.dpde.es $>$.

Polanco Martínez, Fernando. "Redes polisémicas y niveles de interpretación: representación semántica de unidades lingüísticas complejas: el caso de vamos". Estudios de Lingüística Universidad de Alicante 27 (2013): 199-249.

Pons Bordería, Salvador. "La combinación de marcadores del dicurso en la conversación coloquial: interracciones entre posición y función”. Estudos Linguísticos/Linguistic Studies 2 (2008): 141-59.

Portolés, José. Marcadores del discurso. Barcelona: Ariel, 1998.

Ruiz Gurillo, Leonor. "Las locuciones marcadoras del español: análisis y aplicaciones”. Fraseología contrastiva: con ejemplos tomados del alemán, español, 
francés e italiano. Eds. Ramón Almela Pérez, Gerd Wotjak y Estanislao Ramón Trives. Universidad de Murcia: Servicio de Publicaciones, 2005. 241-58.

Santos Río, Luis. Diccionario de partículas. Salamanca: Luso-Española de ediciones, 2003.

Tanghe, Sanne. Marcadores derivados de verbos de movimiento: una aproximación cognitiva a su polifuncionalidad. Berlin: De Gruyter Mouton, 2016.

Traugott, Elizabeth Closs y Richard B. Dasher. Regularity In Semantic Change. Cambridge: Cambridge UP, 2007.

Val.Es.Co: Antonio Briz Gómez y Grupo Val.Es.Co. Corpus de conversaciones coloquiales. Madrid: Arco Libros, 2002.

Waltereit, Richard. "À propos de la genèse diachronique des combinaisons de marqueurs: L'exemple de bon ben et enfin bref”. Langue française 157.2 (2007): 94-109. 